\title{
A Methodology for the Generation of Dynamic Compact Models of Packages and Heat Sinks from Thermal Transient Measurements
}

\author{
M. Rencz ${ }^{1}$, G.Farkas ${ }^{1}$, A. Poppe ${ }^{1}$,V. Székely ${ }^{2}$, B.Courtois ${ }^{3}$ \\ MicReD, Gulyás u 27, H-1112 Budapest, Hungary, rencz@micred.com \\ BUTE Goldmann tér 3 H-1521 Budapest, Hungary, szekely@eet.bme.hu \\ TIMA Laboratoire, 46 av. Felix Viallet,38032 Grenoble, France, courtois@imag.fr
}

\begin{abstract}
In this paper we present a methodology that we have recently elaborated for the generation of transient compact models of packages and heat sinks entirely from measured thermal transient results. The main advantage of generating the models from measured results is the time-gain. We do not need to build up the detailed structural model of the package or the heat sink in order to simulate it, as suggested by the DELPHI methodology. An additional advantage is that the lengthy transient simulations are not needed. In the paper we first summarize the way of generating the compact models of packages and heat sinks from measurements. After this we present how to use the obtained dynamic compact package models in board level simulators, that are extended with the feature of calculating with compact models.
\end{abstract}

\section{Introduction}

Compact models of packages can be very useful substitutes of the detailed models in various simulations. There are now board level simulators available that can simulate the behavior of printed circuit boards populated with packages, where any of these can be substituted by the compact models of their steady state or time dependent thermal behavior [1]. Compact models can be used also to substitute the packages in CFD simulations.

Creation of boundary condition independent compact models is a subject of dispute in the scientific literature for several years now, since the milestone paper of Lasance et al [2], which has presented a method that, became later known as the DELPHI methodology.

This methodology was elaborated for steady state representation of the packages only. It became extended with the procedure of adding capacitances to the steady state model in [4].

The main ideas of the DELPHI methodology are simulation, measurement, data fitting and optimization. To obtain data about the behavior of the package at various boundary conditions first a detailed structural model of the package has to be created. This detailed model has to be simulated by a field solver. The appropriate set of boundary conditions for the simulation was defined in [2]. The measured results have to be validated with and fitted to the measured results such, that some of the boundary conditions are used for the model fitting, some others are used for the validation of the model [3]. The compact model is constructed of a few thermal resistors and capacitors. The structure of this model is the descendent of the detailed model: in first approximation terminals or ports are needed everywhere where boundary conditions are applied in the detailed model, but the optimization procedure may simplify the structure considerably.

The big advantage of the DELPHI methodology is that it delivers relatively accurate compact models for all boundary conditions. Especially the junction temperature accuracy is boundary condition independently good. But the creation of these models requires the generation of a detailed physical model that is rather time consuming in case of complex structures. This is why in spite of all the advantages of the DELPHI method several other methods are appearing in the literature continuously, some of them have been obtaining really big attention like [5], or [6].

A group of the alternative methods wishes to eliminate the need of building the detailed model and the simulation. A typical representative is presented in [7]. The problem of this entirely measurement-based method is that the evaluation algorithm requires highly accurate measurement results, which is in general not easy to obtain.

In our recently developed methodology we combine the optimization strategy of the DELPHI methodology with the idea of using only measured results for the generation of the boundary condition independent dynamic compact models (DCMs) of [7].

\section{The suggested methodology for DCM generation}

In this method we try to keep all the advantages of the DELPHI method, while dropping the disadvantage: the need for detailed model generation, and the need for the extremely time consuming thermal transient simulation, and the need for model validation and model fitting.

The steps of our methodology are as follows:

1. First we obtain all the transient curves that are needed for the optimization from high accuracy and highresolution thermal transient measurements. [8] For these measurements we apply a limited subset of the boundary conditions defined by the DELPHI methodology. The applied boundary conditions are the same that were defined and recommended by the DELPHI methodology for the validation of the simulated detailed model of the package: the double cold plate measurements defined as DCP1, DCP2, DCP3 and DCP4 in [3], see Table 1.

2. We use a further developed version of the DOTCOMP tool described in [3] for fitting compact models to these measurements.

3. We verify the compact models with another set of measurements with new boundary conditions. 
The most critical part of the procedure is the verification of the measurements with new boundary conditions, since the measurements that are easy to realize are only those DCP1, DCP2, DCP3 and DCP4 measurements that were used for the generation of the model. The realization of new, well-defined and reproducible boundary conditions is not easy in practice.

Table 1. The prescribed heat transfer coefficient values in the dual cold plate measurements

\begin{tabular}{|l|l|l|l|l|}
\hline & $\begin{array}{l}a_{\text {lop }} \\
{\left[\mathrm{W} / \mathrm{m}^{2} \mathrm{~K}\right]}\end{array}$ & $\begin{array}{l}\alpha_{\text {bottom }} \\
{\left[\mathrm{W} / \mathrm{m}^{2} \mathrm{~K}\right]}\end{array}$ & $\begin{array}{l}\alpha_{\text {side }} \\
{\left[\mathrm{W} / \mathrm{m}^{2} \mathrm{~K}\right]}\end{array}$ & $\begin{array}{l}\mathrm{A}_{\text {leads }} \\
{\left[\mathrm{W} / \mathrm{m}^{2} \mathrm{~K}\right]}\end{array}$ \\
\hline$\overline{D C P}-1$ & 10000 & 10000 & 1 & 1 \\
\hline$D C P-2$ & 10 & 10000 & 1 & 1 \\
\hline$D C P-3$ & 10000 & 10 & 1 & 1 \\
\hline$D C P-4$ & 1 & 1 & 1 & 10000 \\
\hline
\end{tabular}

A possible solution is to connect well-defined heat transfer coefficient values to the various package surfaces by various heat sinks. The disadvantage of the method is that we need a series of heat sinks that have to be connected, mounted, dismounted to the package, which makes the measurement unreliable. A better solution is to connect controllable thermal resistances to the surfaces by the method presented in [9], see Figure 1. In this active heat $\operatorname{sink}$ realization the current of the Peltier cell is controlled such, that the ratio of the temperature drop on the Peltier cell and the heat flux crossing the cell is kept at a prescribed constant value. This way an electronically adjustable constant heat resistance is realized between the device and the ambience.

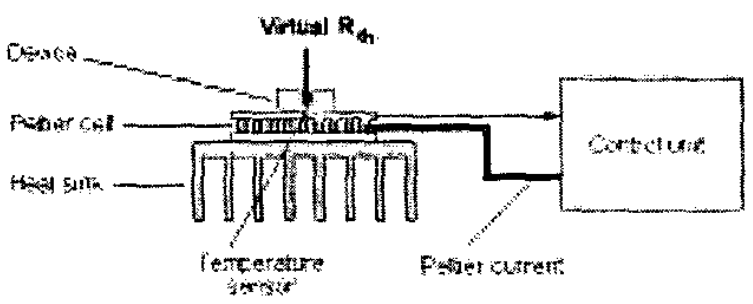

Figure 1 Active heat sink arrangement: an electronically controlled thermal resistance

The main advantage of this solution is that with the same measurement arrangement several $\alpha$ heat transfer coefficients, or $R_{t h}$ values can be realized between the package and the cooling mount, that is, several thermal boundary conditions can be realized without dismounting the measurement arrangement, increasing strongly the reliability of measurement.

A pragmatic idea for the verification measurements is presented in [10]. This suggests finding the compact model of a heat sink with the same measurement and optimization method that is followed for the package. After this connect the heat sink to the package, apply the same set of boundary conditions and find the compact model of the package + heat sink structure. If the compact model of the combined structure produces the same transient curves as the connected individual compact models of the package and the heat sink, then the compact models are considered verified.

\section{Experimental results}

We present the methodology of creating the dynamic compact model of a SOT-93 package of an NPN bipolar power transistor (BD245), see Figure 2. The characteristic faces of the package were named top, bottom and leads.
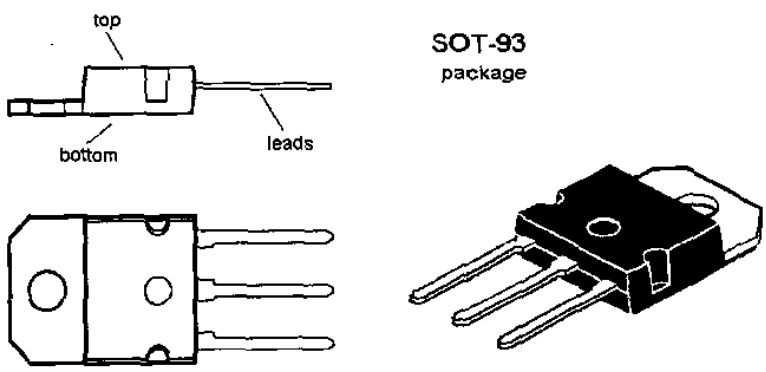

Figure 2 The package to be characterized

For the model generation we used the thermal transient measurement results obtained in the DCP1, DCP2 and DC3 double cold plate arrangements from the DELPHI boundary condition set, (see Table 1). The actual realizations are presented in Figure 3. For the test set we used the transient results of 3 further arrangements: small and large refer to the application of a small and large heat sink on the top of the package, respectively, while the results designated by free refer to the measurement results of the free standing package.

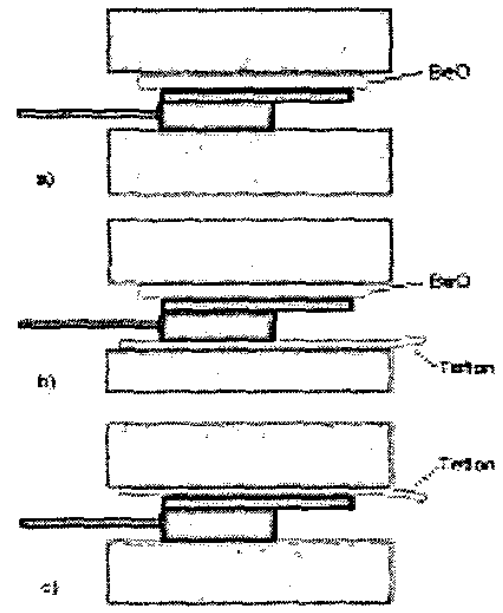

Figure 3 The applied DCP1, DCP2 and DCP3 measurements, respectively

Table 2 The conductors and insulators used in the DCP arrangements of Figure 3 


\begin{tabular}{|c|c|c|c|}
\hline & $\begin{array}{c}\text { thickness } \\
{[\mathrm{mm}]}\end{array}$ & $\begin{array}{c}\text { nominal HTC } \\
{\left[\mathrm{W} / \mathrm{Km}^{2}\right]}\end{array}$ & $\begin{array}{c}\text { Rth }[\mathrm{K} / \mathrm{W}] \\
@ \mathrm{~A}=280\left[\mathrm{~mm}^{2}\right]\end{array}$ \\
\hline BeO & 0.72 & $3.8 \mathrm{E}+05$ & 0.0095 \\
\hline Teflon & 0.19 & $9.1 \mathrm{E}+02$ & 3.9 \\
\hline
\end{tabular}

A power step of $12 \mathrm{~W}$ was applied and $100 \mathrm{sec}$ of the thermal transient was captured real-time, with a high initial time resolution $(1 \mu \mathrm{sec})$ and high temperature resolution, measuring $V_{E B}$ "on-the-fly" as described in [10]. The measurement was followed by a post-processing step in the measurement software, which automatically generates descriptive functions of the packaged device, like the:

- transient response curves

- time constant spectra of these

- cumulative and differential structure functions

The measured transient curves are shown in Figure 4.

We used the DOTCOMP compact model generating tool [3] to optimize the model.

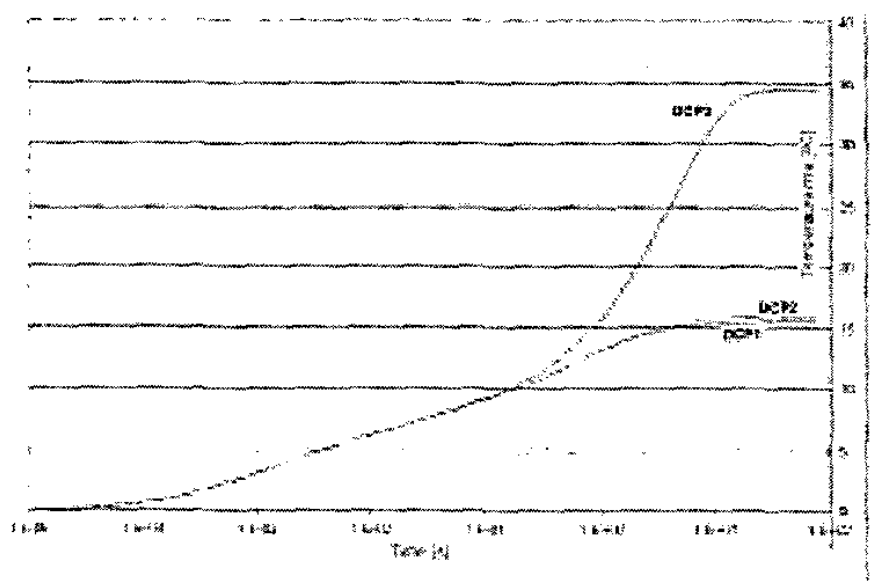

Figure 4 The measured transient curves in the different DCP arrangements

The input data to this program are the heat transfer coefficients on the characteristic faces and the total heat capacitance of the system. We found that the easiest way to create these input data is starting from the so-called structure functions, introduced in [11], and discussed recently in [12]. These are a graphic representation of the one dimensional equivalent detailed thermal RC network of the measured system, see Figure 5, and the heat transfer coefficients, the thermal resistance and the total system capacitance values can be directly read from them.

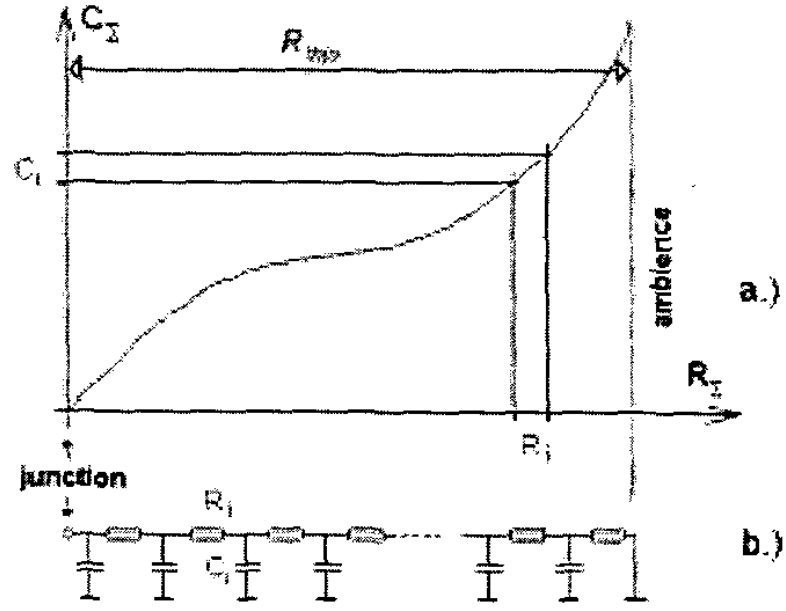

Figure 5 The cumulative structure function of a heat flow path, referring to the partial heat resistances and capacitances, and providing the total $R_{i n}$ of the system

Figure 6 presents the cumulative structure functions derived from the thermal transient measurement results, some of which are presented in Figure 4, and characterizes the thermal conductance path from the junction to the ambience. At low gradient sections a small amount of material having low capacitance causes large change in thermal resistance. These regions have low thermal conductivity or small crosssectional area. Steep sections correspond to material regions of high thermal conductivity or large cross-sectional area. Sudden breaks of the slope belong to material or geometry changes.

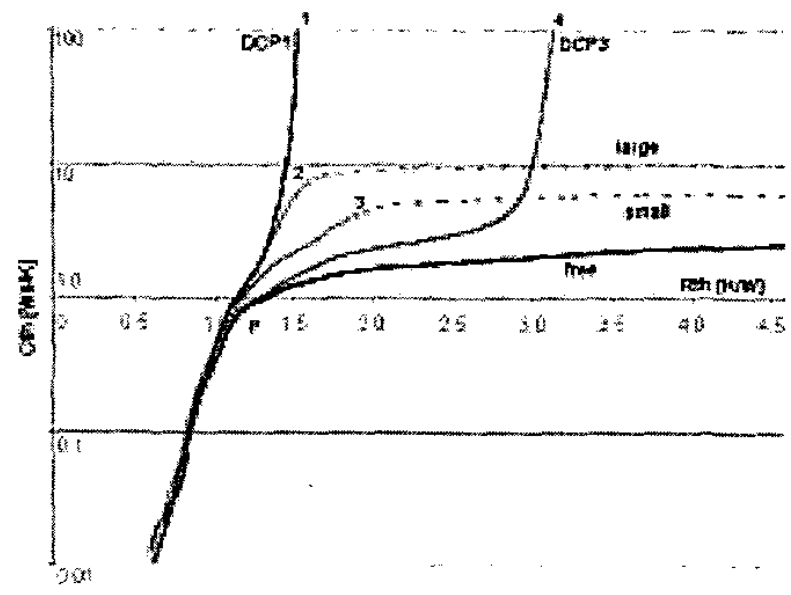

Figure 6 Cumulative structure functions of different thermal measurements on a BD245 transistor. DCPI and $D C P 3$ are the modeling boundary conditions, large, small and free belong to the testing boundary condition set.

It is easier to identify the interface between the sections using the derivative of this function, the differential structure function. In this function the peaks correspond to regions of high thermal conductivity, like a heat sink, and valleys show 
regions of low thermal conductivity, like die attach, see Figure 7.

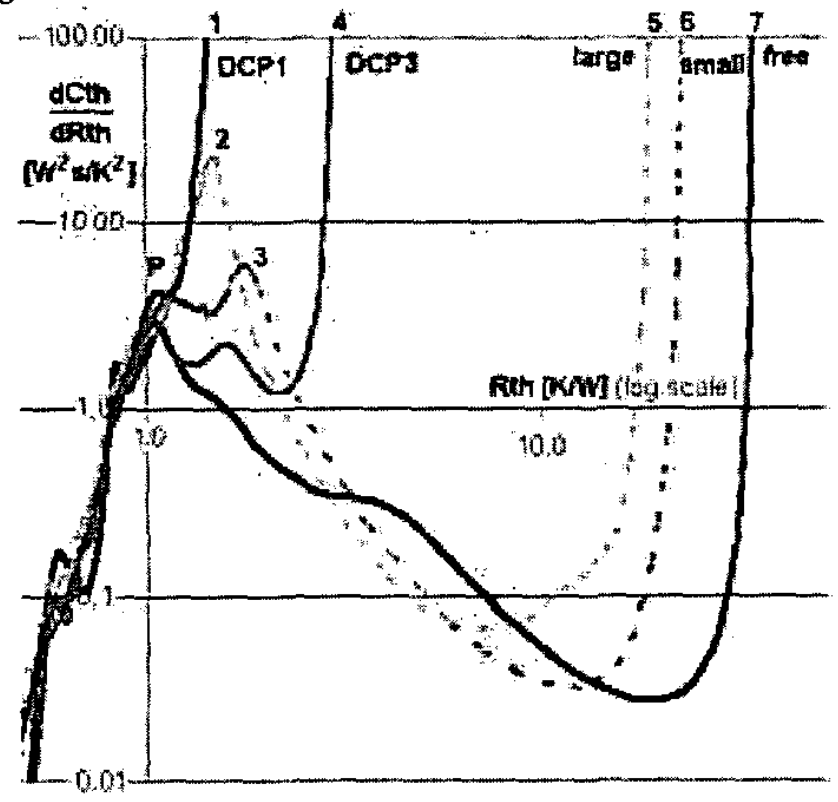

Figure 7 Differential structure functions obtained from the same measurements of the $B D 245$ transistor. $D C P 1$ and $D C P 3$ are the modeling boundary conditions, large, small and free belong to the test boundary condition set.

Characteristic points can be easily identified on the curves, these are points $P$, and $I$ to 7 . All curves nearly coincide from the junction to a peak near point $P$, this is the section of the common heat flow path. In case of this package there is a main heat flow path from the junction towards the copper base, which ends in the bottom face. The peak at $P$ represents the mass of this copper base.

At the ambience the thermal capacitance grows to infinity, the position of 1 and 4 shows the junction-to-ambience values for DCP1 and DCP3.

From the $\mathrm{P}$ point we obtain $R_{t h P}=1.1 \mathrm{~K} / \mathrm{W}$, and a cumulated capacitance for the whole package of $C_{t h P}=0.9$ $\mathrm{W}^{2} \mathrm{~s} / \mathrm{K}^{2}$. The distance of $P$ to $l$ along the $x$ axis gives the case-to-cold-plate thermal resistance for DCP1 which is $R_{t h I}=0.45 \mathrm{~K} / \mathrm{W}$, and about the same is obtained for DCP2. The $P$ to 4 distance gives $R_{t h 3}=2.0 \mathrm{~K} / \mathrm{W}$ for DCP3. From the material parameters and the physical dimensions we can calculate the $\alpha$ effective heat transfer coefficients. The bottom face is approximately $280 \mathrm{~mm}^{2}$, and the top face is approximately $200 \mathrm{~mm}^{2}$, consequently from $R t h_{l}$ and $R t h_{2}$ we get an $\alpha$ of $8000 \mathrm{~W} / \mathrm{Km}^{2}$ towards one side of the cold-plate. This is much below the $\alpha$ heat transfer coefficient value represented by the $\mathrm{BeO}$ ceramics, so this value really shows the heat removal capability of the cold-plate.

The DOTCOMP program starts with the generation of the steady state model, and extends it to a dynamic model by adding capacitances to it, according to the procedure suggested in [4]. For the steady state model it assumes the model topology suggested in [3] and shown in Figure 8.

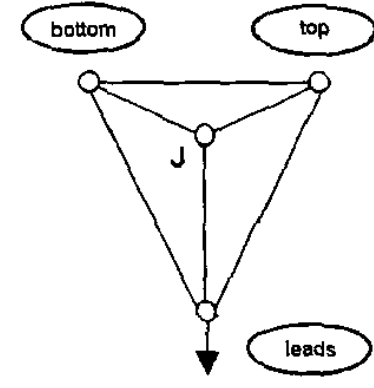

Figure 8 The initial model topology suggested by the DOTCOMP tool, $J$ refers to the junction

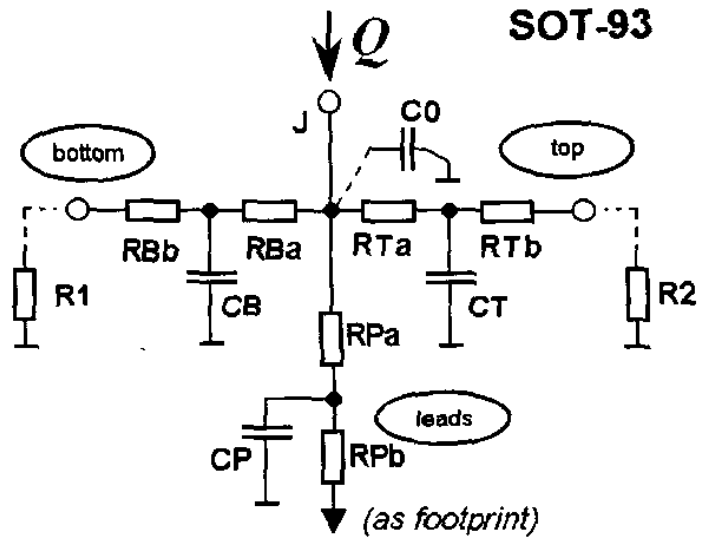

Figure 9 The dynamic compact model optimized by DOTCOMP for the SOT-93 package from the measured data

Table 3 The element values of Figure 9

\begin{tabular}{|c|c|c|c|c|}
\hline & & $\mathbf{R 1}[\mathrm{K} / \mathrm{W}]$ & $\mathbf{R 2}[\mathrm{K} / \mathrm{W}]$ & \\
\hline in DCP1 & & 0.45 & 0.45 & \\
\hline in DCP3 & & 4.35 & 0.45 & \\
\hline & $\boldsymbol{x}$ & $\mathbf{R} x \mathbf{a}[\mathrm{K} / \mathrm{W}]$ & $\mathbf{R} x \mathbf{b}[\mathrm{K} / \mathrm{W}]$ & $\mathbf{C} x\left[\mathrm{~W}^{2} \mathrm{~s} / \mathrm{K}^{2}\right]$ \\
\hline bottom & $\mathrm{B}$ & 1.25 & 0.05 & 2.1 \\
\hline top & $\mathrm{T}$ & 0.9 & 6.1 & 0.1 \\
\hline leads & $\mathrm{P}$ & 1.05 & 17 & 0.005 \\
\hline "chip" & 0 & & & 0.002 \\
\hline
\end{tabular}

The tool first requires the steady-state temperatures of the model set and the $\alpha$ values, represented by the R1 and R2 resistors in Figure 9. The $\alpha$ values for the leads resistor were not obtained from DCP4 type (leads only) measurement, as suggested by the DELPHI methodology, since it was easy to calculate it from the wire geometry.

As a result of the optimization process we obtained that the face-to-face resistances are high and can be omitted, see Figure 9, as we expected for this package structure. For the resistor values we got $R_{B}=1.3 \mathrm{~K} / \mathrm{W}, R_{T}=7.0 \mathrm{~K} / \mathrm{W}, R_{p}=18.05$ $\mathrm{K} / \mathrm{W}$.

These values were subdivided in the transient optimization process in two parts, giving the optimal locations for the capacitors for the best fit of the transient curves. The transient

2003 IEEE/CPMT/SEM] Int'I Electronics Manufacturing Technobogy Symposium 
curve fitting was not extended to the time range below $0.1 \mathrm{~ms}$, $\mathrm{CO}$ was added manually for model consistency at short times.

The comparison of the measured curves and the ones calculated with the dynamic compact model by the board level solver of [1] is presented in Figure 10, where the applied power was $12 \mathrm{~W}$, dcplm and dcp3m are the measured and dcpls and dcp3s are the computed curves.

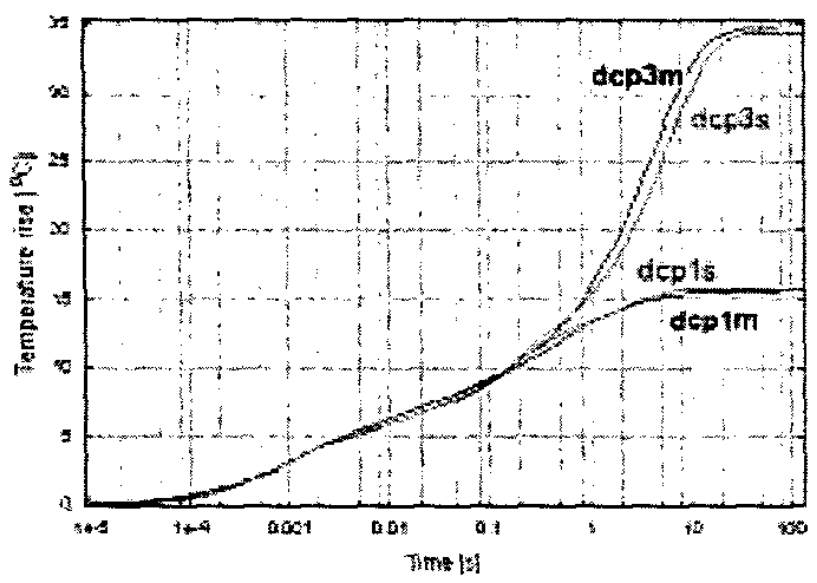

Figure 10 Comparison of measured (dcp Im, dcp $3 m$ ) and simulated (dcpls, dcp3s) thermal transient curves

A characteristic feature of compact modeling is presented in Figure 11: the compact models provide a good approximation of the behavior of the modeled system, but with the limited number of time constants of the DCM model the curves can not be as smooth as the ones they approximate.

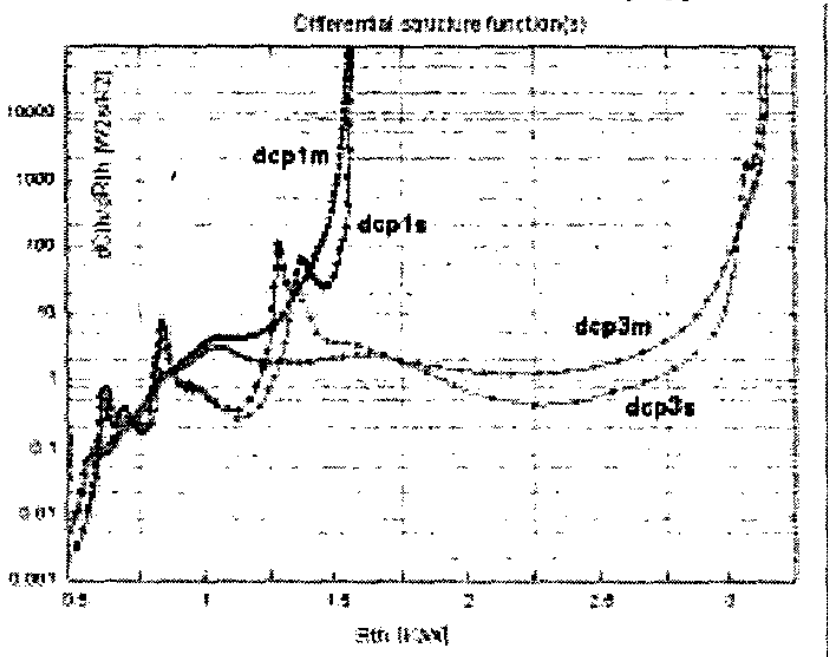

Figure 11 differential structure functions calculated from the measured and simulated curves of Figure 10

The presented methodology can be followed for the generation of dynamic compact models of heat sinks as well. In case of heat sinks the use of a small transistor is recommended that we use for powering and measuring the temperature of the heat sink [10].

If we have heat sinks with known compact models we can validate the package compact models with new measurement, that are compared to new simulation results. In the new measurements we use the heat sinks as new boundary conditions to the package. These thermal transient curves have to be validated with the simulated results of the package + heat sink combined DCM that is obtained by the direct connection of the appropriate terminals of the compact models of the package and the heatsink.

The results of this comparison are presented in Figure 12. To obtain these curves we used a very simple model for the heat sinks: a parallel $R_{t h} C_{t h}$ pair between the thermal ground and the sink surface node is connected with an $R_{t h i}$ element, representing the interface thermal resistance, to the package surface node. Such a simple model is shown in Figure 13 and Figure 14, where the actual value of $R_{t h i}=0$.

Note, that the agreement of the measured and simulated curves for the package + heat sink combined structure is very good for the case of the large heat sink, which shows that the package model is good. The small difference in the measured and simulated curves in case of the package + small heat sink arrangement can be attributed to the improper characterization of the small heat sink with the very simple model.

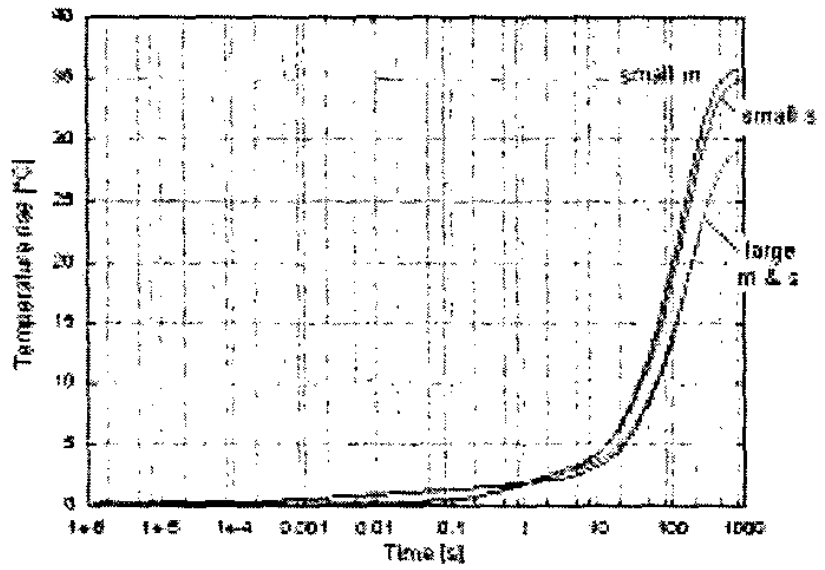

Figure 12 Non-fitted measured and simulated results for the package + heat sink structure, for two different heat sink models, the package model is that of Figure 9

Once we have the methodology of creating dynamic compact package models as well as compact models of cooling assemblies, we can create model libraries for thermal simulators that can co-simulate the detailed model of a PWB and the attached compact device models, like the THERMAN thermal simulator tool [14].

Figure 13 presents the board- package DCM cosimulation concept of [1]. The board model is populated with packages, some of them represented with their dynamic compact package models and some of these are terminated on their top surface with compact models of cooling mounts. The heat exchange between the packaged devices and the board takes place through the footprints of the packages. Further heat removal to the ambient can be modeled by heat transfer coefficients on the package surfaces (representing e.g. natural convection) or by the compact models of heat sinks, connected to the compact models of the packages . 
In Figure 14 we present a simple model of a PGA package and a cooling mount. The model library entry of the package is completed with the geometry of the footprints such as shown in Figure 15. Figure 16 shows a simple simulation example: a steady-state temperature distribution of a board with two such packages both dissipating $1 \mathrm{~W}$. The package on the right is equipped with a cooling mount, resulting in less heat transferred to the board and lower junction temperatures. The same behavior can be observed on junction temperature transients.

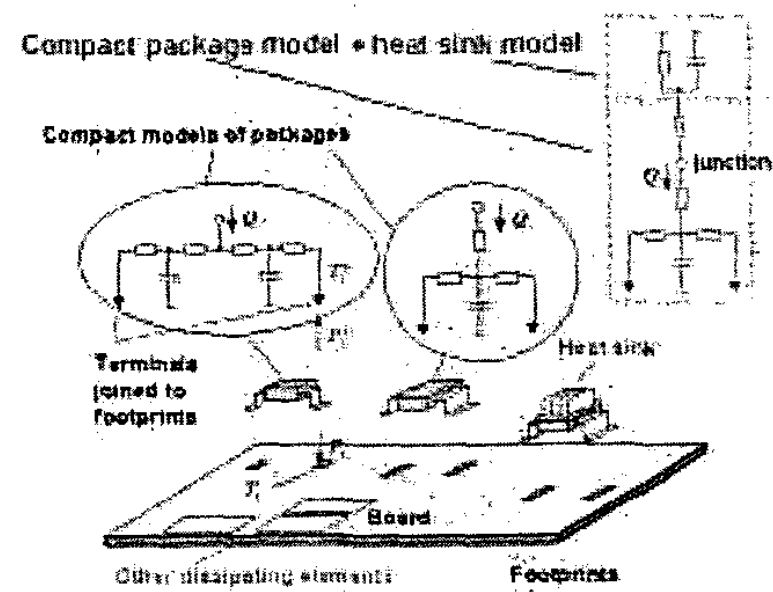

Figure 13 The concept of co-simulation of a detailed board model with dynamic compact models of packages and cooling assemblies such as heat-sinks

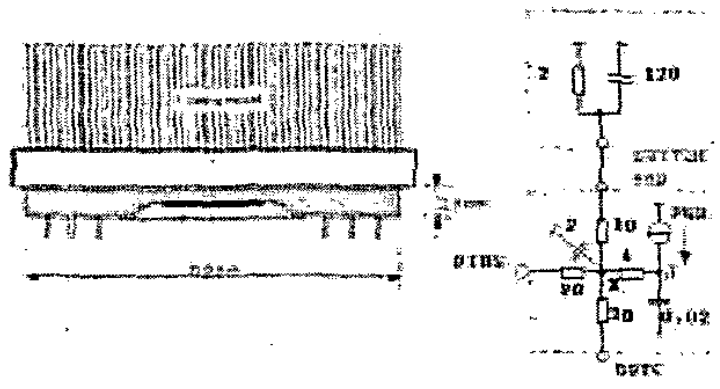

Figure 14 Compact model example of a PGA package and a finned cooling mount

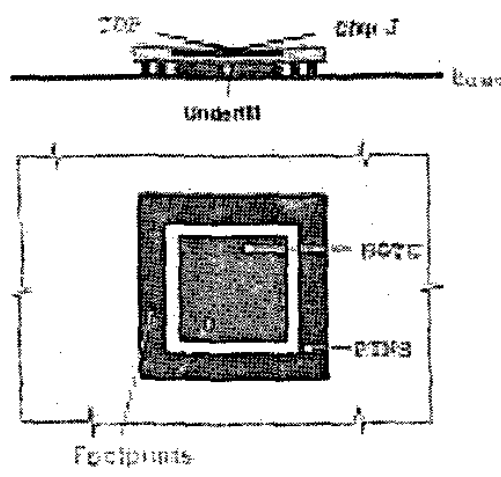

Figure 15 Footprint geometry of the PGA package shown in Figure 14
Note in Figure 17 that the initial parts of the simulated transients coincide for shorter times, when the thermal wave propagates inside the package.

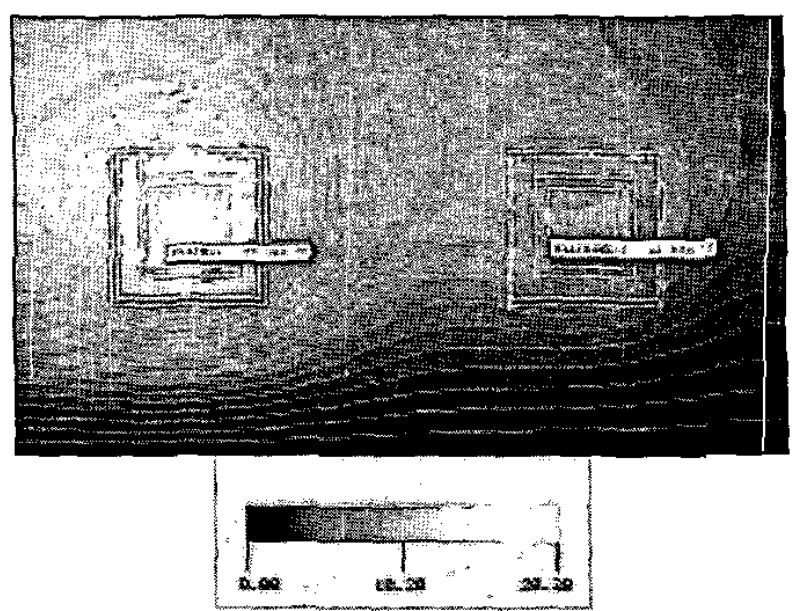

Figure 16 Steady-state temperature distribution of a PWB with two PGA models, and the junction temperatures. The package on the right has an attached heat-sink model as shown in Figure 14.

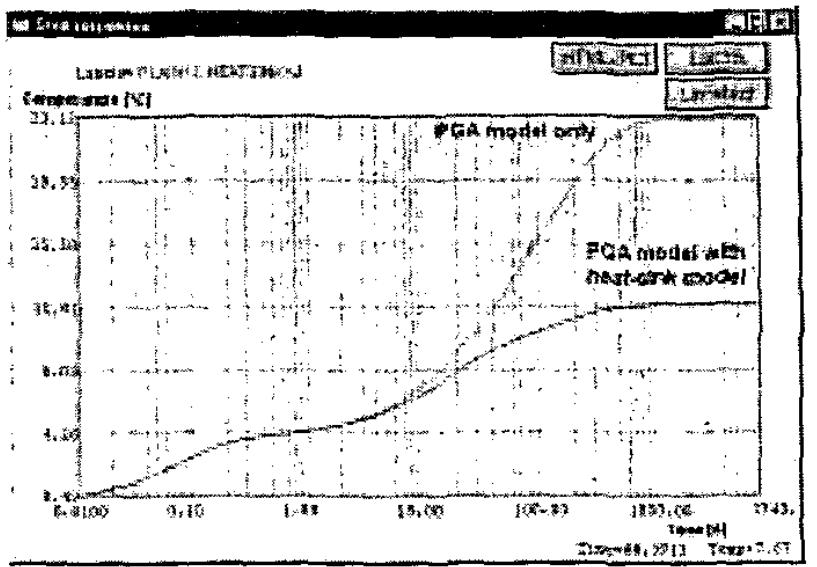

Figure 17 Thermal transient responses obtained by the two models as calculated by the THERMAN program [1], [14].

\section{Conclusions}

We have presented a methodology for creating dynamic compact thermal models of packages and/or cooling mounts entirely from measured thermal transient results. The idea is a derivative of the DEPHI methodology, adapted for convenient time dependent model generation. The main advantage of the presented methodology is that it does not request the creation of the detailed compact model, and the lengthy transient simulation is also substituted by fast transient measurements.

The use of the method is presented on an example: the creation of a dynamic compact model for a SOT93 package is discussed in details. In the creation of the DCP model of the package the measurement and model identification technique was the one developed within the PROFIT project [15].

2003 IEEE/CPMT/SEMI Int'I Ejectronics Manufacturing Technology Symposium 
Models of cooling mounts are easily created similarly, from the structure functions derived by the evaluation of the measured high-resolution thermal transients.

The modeling approach presented here is another example of using structure functions: the major structural elements of the packaged device can be identified from them and the starting element values of a dynamic compact package model can also be found on them. The package models can be finetuned by proper fitting tools. In case of cooling assemblies the element values of a simple but sufficiently accurate compact model can be read directly from the structure functions obtained from measured thermal transient responses.

Attaching compact models of cooling assemblies to compact models of packages increases the accuracy of boardlevel simulators at virtually no cost in terms of CPU time, since during the co-simulation with the detailed model more than $99 \%$ of the CPU power is spent on solving the latter.

\section{Acknowledgments}

This work was supported by the PROFIT IST-1999-12529 Project (Prediction of Temperature Gradients Influencing the Quality of Electronic Products) of the EU and by the INFOTERM $2 / 018 / 2001$ NKFP project of the Hungarian Ministry of Education (OM). The help of E. Kollár in doing some of the measurements acknowledged and thanked.

\section{References}

1. M. Rencz, V. Székely, A. Poppe, B. Courtois: Cosimulation of dynamic compact models of packages with the detailed models of printed circuit boards, SEMICON West 2002, July 16-18 2002, San Jose, CA, USA, Proceedings pp 285-290

2. C. Lasance, H. Vinke, H. Rosten, K. $-L$ Weiner: A Novel Approach for the Thermal Characterization of Electronic Parts, Proc. of SEMITHERM XI, San Jose, CA, pp. 1-9. 1995

3. C. Lasance, D. den Hertog, P. Stehouwer : Creation and Evaluation of Compact Models for Thermal Characterisation Using Dedicated Optimisation Software, Proc. of SEMITHERM XV, San Diego, CA, pp. 189-200, 1998

4. F. Christiaens, B. Vandevelde, E. Beyne, R. Mertens, J. Berghmans: "A Generic methodology for Deriving Compact Dynamic Thermal Models, Applied to the PSGA package" IEEE Trans. On Components, Packaging and Manufacturing Technology, Part A Vol 21, No.4 Dec.1998 pp 565-576

5. A. Bar-Cohen and W.B. Krueger: "Thermal characterization of chip packages - evolutionary development of compact models", SEMI-THERM'97, 28-30 January, 1997, Austin, USA, Proceedings pp. 180-197

6. Bosch E., 2001, Thermal Compact Models: An Alternative Approach, Proc. $7^{\text {th }}$ THERMINIC Workshop, pp. 191-196

7. M. Rencz, V. Székely: Dynamic thermal multiport modeling of IC packages. IEEE Transactions on Components and Packaging Technologies, Vol.24, No 4, Dec.2001,pp 596-604
8. V. Székely, M. Rencz: Increasing the accuracy of thermal transient measurements, IEEE Transactions on Components and Packaging Technology, Vol 25, No 4, Dec. 2002, pp

9. V. Székely, A. Nagy, S. Török, G. Hajas, M. Rencz: Realization of an electronically controlled thermal resistance. Microelectronics Journal, Vol 31, No10, pp $727-734,2000$

10. G. Farkas, A,Poppe, E.Kollar, P.Stehouwer: Dynamic compact models of cooling mounts for fast board level design, Proc. of the $19^{\text {th }}$ SEMITHERM, San Jose, CA, USA, March 11-13,2003, pp 255- 262

11. A. Poppe, V. Székely: Dynamic Temperature Measurements: Tools Providing a Look into Package and Mount Structures, Electr. Cooling Magazin, Vol.8, No.2, May 2002.

12. V. Székely and Tran Van Bien: "Fine structure of heat flow path in semiconductor devices: a measurement and identification method", Solid-State Electronics, Vol. 31, pp.1363-1368 (1988)

13. M. Rencz, V. Székely, A. Morelli, C. Villa: Determining partial thermal resistances with transient measurements and using the method to detect die attach discontinuities, SEMITHERM, March 1-14 2002, San Jose, CA,USA, Proceedings pp 15-20

14. V. Székely, A. Poppe, M. Rencz, M. Rosental, T. Teszéri: THERMAN: a thermal simulation tool for IC chips, microstructures and PW boards. Microelectronics Reliability, Vol. 40, pp. 517-524, 2000 .

15. H. Pape et al. : Thermal transient modeling and experimental validation in the European project PROFIT, Proc. of the $19^{\text {th }}$ SEMITHERM, San Jose, CA,USA, March 11-13,2003, pp 247- 254 